\title{
Hot Air Generator Using Natural Convection Flow in a Heated Channel
}

\author{
Bushra AlGarawi and Zeinebou Yahya \\ Department of Physics, Faculty of Sciences, Qassim University, Buraidah 51452, Kingdom of Saudi Arabia
}

\begin{abstract}
Hot air producing is one of the most important engineering applications in recent years. It is a technique used in various thermodynamic systems, such as home heating systems, food dryers. One of the main problems impeding the spread of hot air producing technology is the lack of homogeneity of the heat flow coming from hot air generators as well as an inadequate flow rate. The most of the existing hot air generators require to be supported by systems that can increase the low volumetric flow and the air temperature of these generators, through increasing the speed of the flow of air emitted or lifting the drawer Heat, which contributes to raising the overall cost. However, to improve the thermal and dynamic quality of the hot air flow produced by the generator, a numerical investigation of the free convection flow inside two different configurations is presented in this thesis. The primary objective of this work is to predict the behavior of the flow inside tow configurations, the first one consists of a vertical cylinder with heated walls, and the second configuration is an open-ended vertical cylinder with a hot disc placed at the entrance (configuration A, configuration B). This work characterizes through the examination of this flow, the variables that control an air emission with high flow rate and a high and homogeneous temperature to represent the appropriate criteria that should be respected to obtain a hot air generator overcoming the previously mentioned constraints. Furthermore; the results of this work show the influence the boundary conditions and Rayleigh number on the resulting flow.
\end{abstract}

Key words: Hot air generator, natural convection, vertical channel, Navier-Stokes equations, finite volume method.

\section{Introduction}

The production of hot air has become an indispensable process of everyday life such as drying granular products, heating systems, cooking and food industry.

One of the main problems impeding the spread of hot air producing technology is the lack of homogeneity of the heat flow coming from hot air generators as well as an inadequate flow rate.

The improvement of the quality of the hot air generator became a rich subject of academic and industrial research. Although some experimental studies relating to hot air generator have been conducted $[1,2]$, there is, however, a crucial lack of numerical investigations on hot air generator.

In this thesis, the aim is to numerically simulate the natural convection flow caused by buoyancy forces due

Corresponding author: Zeinebou Yahya, Ph.D., assistant professor, research fields: numerical modeling (CFD) and heat transfer. to the variations in density of temperature, in different configurations. This way one can get sufficient conditions for a natural hot air generator with quasi-uniform temperature and velocity for multiple utilizations. The flow behavior is investigated in a vertical channel with heated walls, and in an open-ended vertical cylinder with a generating source centered at the entrance of the cylinder (Fig. 1).

In this work the flow is modeled mathematically by Navier-Stokes equations, taking into account the differences between the boundary conditions of the studied configurations (mathematical modeling), then finding dynamic and thermal fields of the air emitted from the hot air generator by numerically solving the equations which represent the studied flow (numerical modeling), finally, changing a number of the effective variables of the flow and comparing their associated results, to obtain the criteria that ensure the most suitable solutions for the disadvantages of hot air generators. 


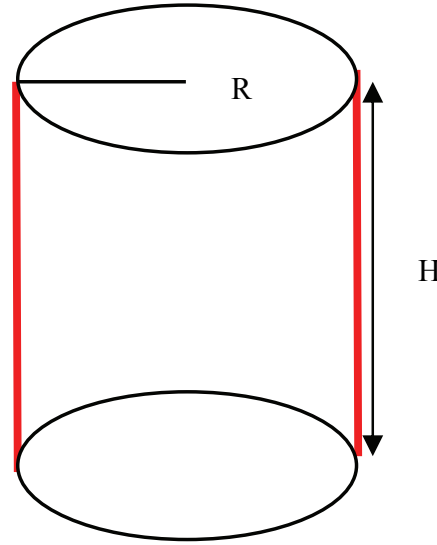

(a)

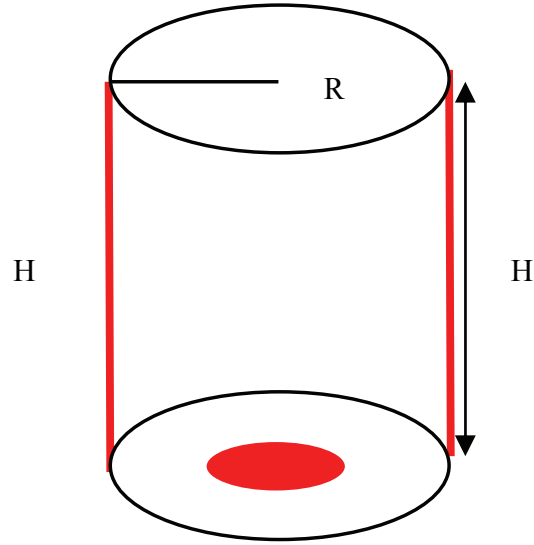

(b)

Fig. 1 Configuration studied: (a) Vertical channel with heated walls; (b) Open-ended vertical cylinder with a generating source centered at the entrance of the cylinder.

\section{Theoretical Model}

The governing equations for steady, laminar, two-dimensional, natural convection flow using Boussinesq approximation are continuity equation, Navier-Stokes equations, and energy equation which are written in cylindrical coordinates as:

$$
\begin{gathered}
\frac{\partial v_{r}}{\partial r}+\frac{v_{r}}{r}+\frac{\partial v_{z}}{\partial z}=0 \\
\left(v_{r} \frac{\partial v_{r}}{\partial r}+v_{z} \frac{\partial v_{r}}{\partial z}\right) \\
=-\frac{1}{\rho_{0}} \frac{\partial p_{m}}{\partial r} \\
+v\left[\frac{\partial}{\partial r}\left(\frac{1}{r} \frac{\partial}{\partial r}\left(r v_{r}\right)\right)+\frac{\partial^{2} v_{r}}{\partial z^{2}}\right] \\
\left(v_{r} \frac{\partial v_{z}}{\partial r}+v_{z} \frac{\partial v_{z}}{\partial z}\right) \\
=-\frac{1}{\rho_{0}} \frac{\partial p_{m}}{\partial z} \\
+v\left[\frac{1}{r} \frac{\partial}{\partial r}\left(r \frac{\partial v_{z}}{\partial r}\right)+\frac{\partial^{2} v_{z}}{\partial z^{2}}\right] \\
+\mathrm{g} \beta_{0}\left(T-T_{0}\right) \\
\rho_{0} c_{p}\left\{v_{r} \frac{\partial T}{\partial r}+v_{z} \frac{\partial T}{\partial z}\right\} k\left[\frac{1}{r} \frac{\partial}{\partial r}\left(r \frac{\partial T}{\partial r}\right)+\frac{\partial^{2} T}{\partial z^{2}}\right]
\end{gathered}
$$

The governing equations are differential equations. Therefore, the boundary conditions must be determined to get the exact solutions for the equations [3].
The boundary conditions for a channel without heating source are as follows:

- At the wall: $r=R$, no-slip boundary condition $v_{r}(R, z)=v_{z}(R, z)=0 \quad$ and $T(R, z)=T_{\mathrm{w}}$.

- At the center: $r=0, \frac{\partial v_{r}(0, z)}{\partial r}=\frac{\partial v_{z}(0, z)}{\partial r}=0$ and $\frac{\partial T(0, z)}{\partial r}=0$.

- At the entrance: $z=0, v_{r}(r, 0)=\frac{\partial v_{z}(r, 0)}{\partial z}=0$ and $T(r, 0)=T_{0}$.

- At the outlet: $z=h, \frac{\partial v_{r}(r, h)}{\partial z}=\frac{\partial v_{z}(r, h)}{\partial z}=$ $\frac{\partial p(r, h)}{\partial z}=0$ and $\frac{\partial T(r, h)}{\partial z}=0$.

Same boundary conditions are applied to the channel with a heating source except the entrance boundary conditions [4] where:

- At $0 \leq r \leq R_{s}, v_{z}(r, 0)=v_{r}(r, 0)=V_{0}$

$T(r, 0)=T_{s}$.

- At $R_{s} \leq r \leq 0.5 A, v_{r}(r, 0)=\frac{\partial v_{z}(r, 0)}{\partial z}=0$ and $T(r, 0)=T_{0}$.

where $R_{S}$ is the rayon of the heating source and $A$ is the form factor.

In order to make the governing equations easier to solve, they are written in their non-dimensional form by substituting the following non-dimensional 
parameters [5]:

$$
\begin{gathered}
\theta=\frac{T-T_{0}}{T_{w}-T_{0}} \\
r^{*}=\frac{r}{h} \\
z^{*}=\frac{z}{h} \\
V_{r}=\frac{v_{r}}{V_{0}}=\frac{v_{r} h}{\alpha_{0}} \\
V_{z}=\frac{v_{z}}{V_{0}}=\frac{v_{z} h}{\alpha_{0}} \\
P=\frac{p_{m}}{P_{0}}=\frac{p_{m}}{\rho V_{0}^{2}}
\end{gathered}
$$

Thus, the governing equations in their non-dimensional form are:

$$
\begin{aligned}
& \frac{\partial V_{r}}{\partial r^{*}}+\frac{V_{r}}{r^{*}}+\frac{\partial V_{z}}{\partial z^{*}}=0 \\
& \left(V_{r} \frac{\partial V_{r}}{\partial r^{*}}+V_{z} \frac{\partial V_{r}}{\partial z^{*}}\right) \\
& =-\frac{\partial P}{\partial r^{*}} \\
& +\operatorname{Pr}\left[\frac{1}{r^{*}} \frac{\partial V_{r}}{\partial r^{*}}+\frac{\partial^{2} V_{r}}{\partial r^{* 2}}+\frac{\partial^{2} V_{r}}{\partial z^{* 2}}\right] \\
& -\operatorname{Pr} \frac{V_{r}}{r^{* 2}} \\
& \left(V_{r} \frac{\partial V_{z}}{\partial r^{*}}+V_{z} \frac{\partial V_{z}}{\partial z^{*}}\right) \\
& =-\frac{\partial P}{\partial z^{*}} \\
& +\operatorname{Pr}\left[\frac{1}{r^{*}} \frac{\partial V_{z}}{\partial r^{*}}+\frac{\partial^{2} V_{z}}{\partial r^{* 2}}+\frac{\partial^{2} V_{z}}{\partial z^{* 2}}\right] \\
& +\operatorname{RaPr} \theta \\
& \left\{V_{r} \frac{\partial \theta}{\partial r^{*}}+V_{z} \frac{\partial \theta}{\partial z^{*}}\right\}=\left[\frac{1}{r^{*}} \frac{\partial \theta}{\partial r^{*}}+\frac{\partial^{2} \theta}{\partial r^{* 2}}+\frac{\partial^{2} \theta}{\partial z^{* 2}}\right]
\end{aligned}
$$

They are written in their conservative form:

$$
\begin{gathered}
\operatorname{div} \vec{V}=0 \\
\operatorname{div}\left[\vec{V} V_{r}-\operatorname{Pr} \overrightarrow{\operatorname{grad}} V_{r}\right]=-\frac{\partial P}{\partial r}-\operatorname{Pr} \frac{V_{r}}{r^{2}} \\
\operatorname{div}\left[\vec{V} V_{z}-\operatorname{Pr} \overrightarrow{\operatorname{grad}} V_{z}\right]-\frac{\partial P}{\partial z}-\operatorname{Ra} \operatorname{Pr} \theta \\
\operatorname{div}[\vec{V} \theta-\overrightarrow{\operatorname{grad}} \theta]=0
\end{gathered}
$$

In the present study, finite volume method will be used [6]. The most important advantage of using the finite volume method is that the flux is conserved. The first step of finite volume technique is dividing the domain into discrete control volumes where each node is surrounded by the control volume.

Solutions are obtained using line-by-line method. Velocity-pressure coupling is resolved using SIMPLER algorithm. Results are found by a FORTRAN code.

\section{Numerical Results and Discussion}

Our results are relative to the air with a number of Prendtl equal to 0.7 , the Rayleigh number is taken at $\mathrm{Ra}=10^{+7}$ and the form factor is equal to 0.3 .

The results are found for two different configurations to investigate the flow behavior in a vertical channel with heated walls, and in an open ended vertical cylinder with a generating source centered at the entrance of the cylinder (configuration A, configuration $\mathrm{B}$ ).

The convergence criterion for all variables is set to $\varepsilon_{F}=10^{-4}$ in the Fortran code.

The mesh $501 \times 501$ is a good compromise between high accuracy and an acceptable volume of computation.

\subsection{Dynamic and Thermal Fields for the Configuration A}

The radial distribution of the longitudinal dimensionless velocity for different stages $Z$ is shown in Fig. 2a. As illustrated by this figure, the axial velocity is important in a region close to the heated wall and it decreases with a decrease of the radial distance $r$.

The existence of the wall indicates a deviation of the flow to which it leads to boundary layer flow in the vicinity of the wall [6].

The radial distribution of the dimensionless temperature for different stages $\mathrm{Z}$ is shown in Fig. $2 \mathrm{~b}$. As shown in this figure, the temperature is found to be important in a region close to the heated wall, and it 


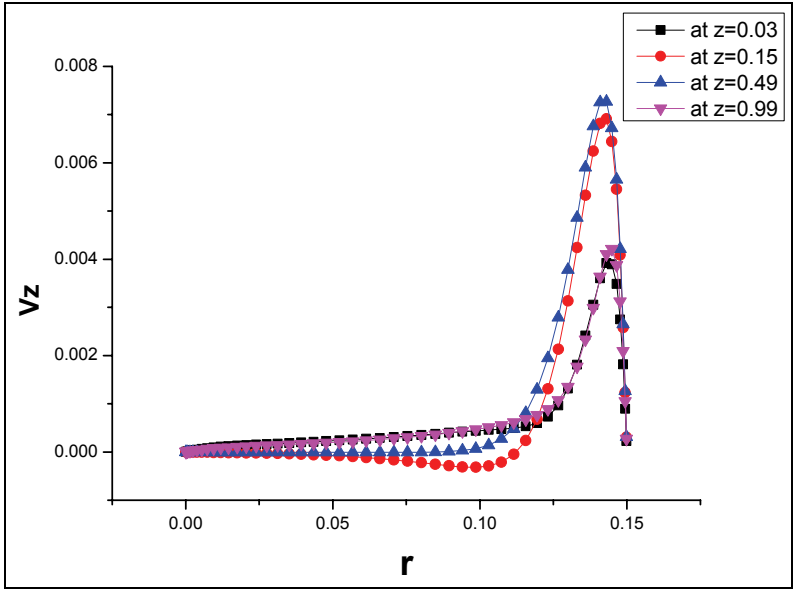

(a)

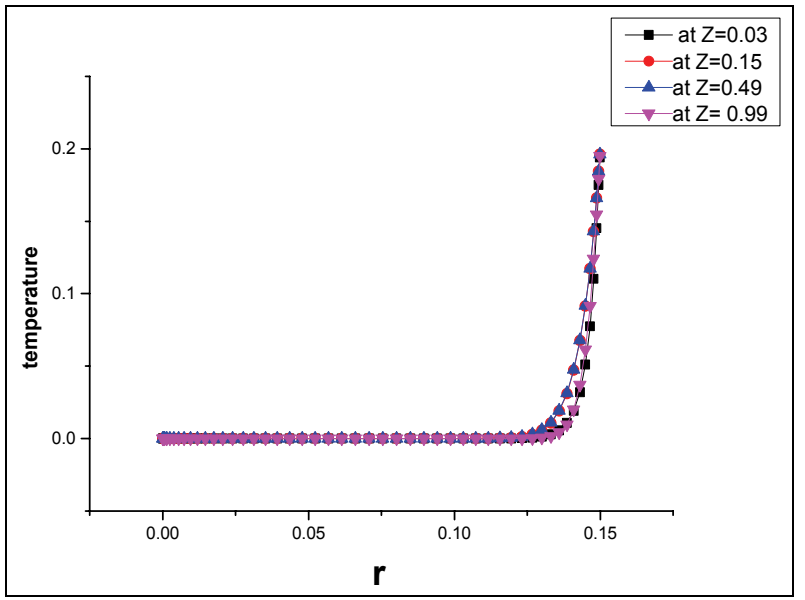

(b)

Fig. 2 Radial distribution of the dynamic and thermal flows for different stages $Z$ : (a) Dimensionless vertical component velocity; (b) Dimensionless temperature.

decreases with a decrease of the radial distance $r$, near the wall of the cylinder an increase of temperature is observed and there is also a flow of thermal boundary layer type.

The profiles become self-similar and the flow can be considered as thermally established.

\subsection{Dynamic and Thermal Fields for the} Configuration $B$

From Fig. 3a, we see that the domain of the velocity in presence of a heating source is divided into three regions. Starting from above the heating source where the velocity increases until $r=0.05$ where the fresh air comes inside the channel, thus the velocity decreases as in the study [2] for an aspect ratio equal to 0.15 and

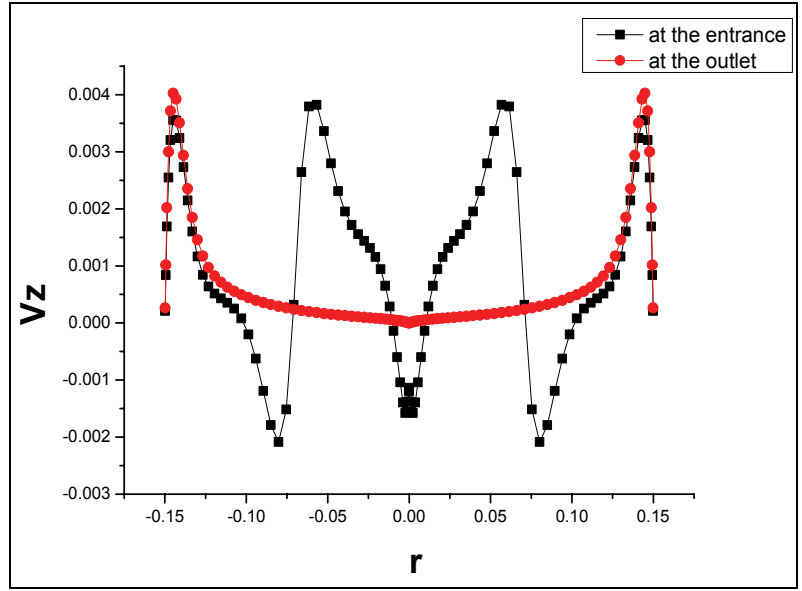

(a)

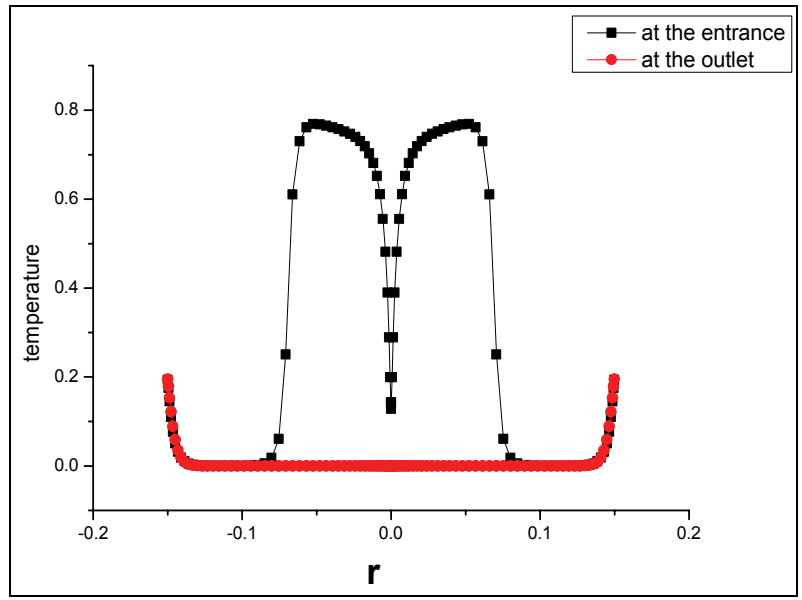

(b)

Fig. 3 Comparison between the profiles at the entrance and the outlet: (a) Dimensionless vertical component velocity; (b) Dimensionless temperature.

a vertical source-cylinder spacing equal to -0.714 . The third region is near the wall where the flow accelerated then decelerated until it reaches zero at the wall. The figure shows a good uniformity of the flow at the exit of the channel [2]. At the outlet of the channel, the flow only accelerates in the region after $r=0.12$ then decelerates until it reaches zero at the wall which gives a velocity peak near the wall.

In Fig. 3b, we notice at the center of the heating source that there is a raise in temperature due to the high temperature of the heating source. At $r=0.05$ the temperature suddenly drops due to the supplement of fresh air coming inside the channel. Then near the wall, the temperature increases again due to the high temperature of the heated wall until it reaches the 
dimensionless temperature which is equal to 0.2 . The figure looks similar to the given in the study [2] for an aspect ratio equal to 0.15 and a vertical source-cylinder spacing equal to -0.714 . The figure shows a good homogenization of the flow at the exit of the channel [2].

It could be seen from Figs. 4 and 5 that the presence of the heating source only affects the flow at the entrance of the channel.

To predict the effect of Rayleigh number on the flow, Fig. 6 shows a comparison between the velocity of the flow for different Rayleigh numbers, at the entrance and the outlet.

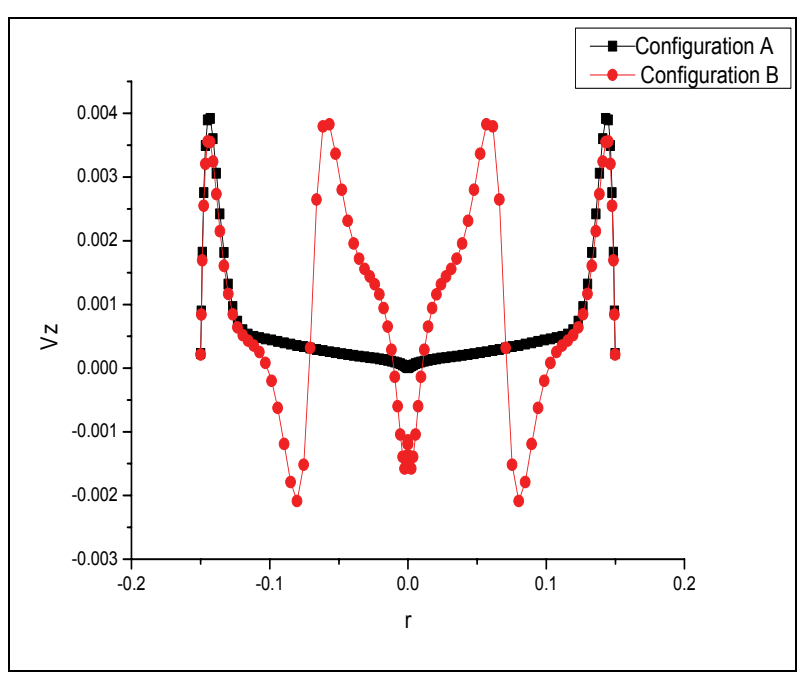

(a)

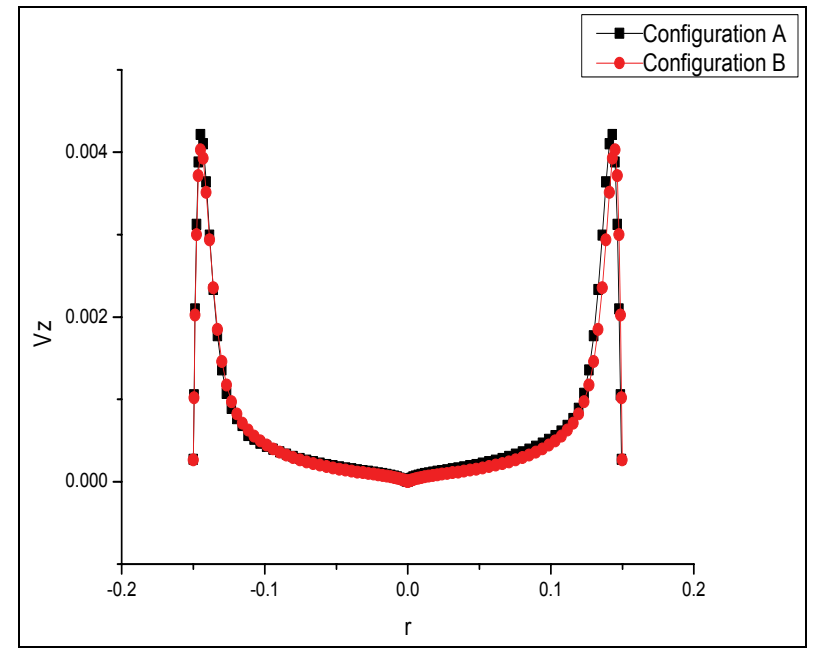

(b)

Fig. 4 Dimensionless vertical velocity profiles for the different configurations: (a) at the entrance; (b) at the outlet.

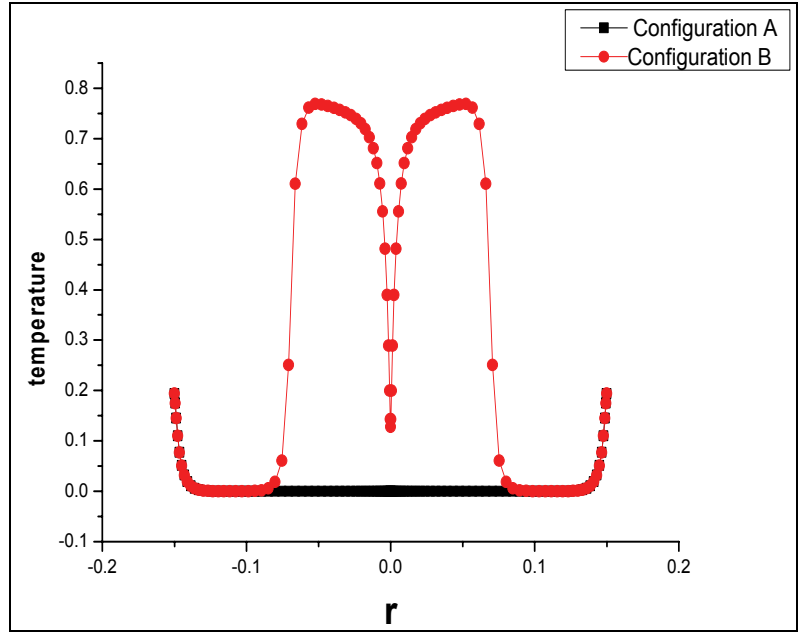

(a)

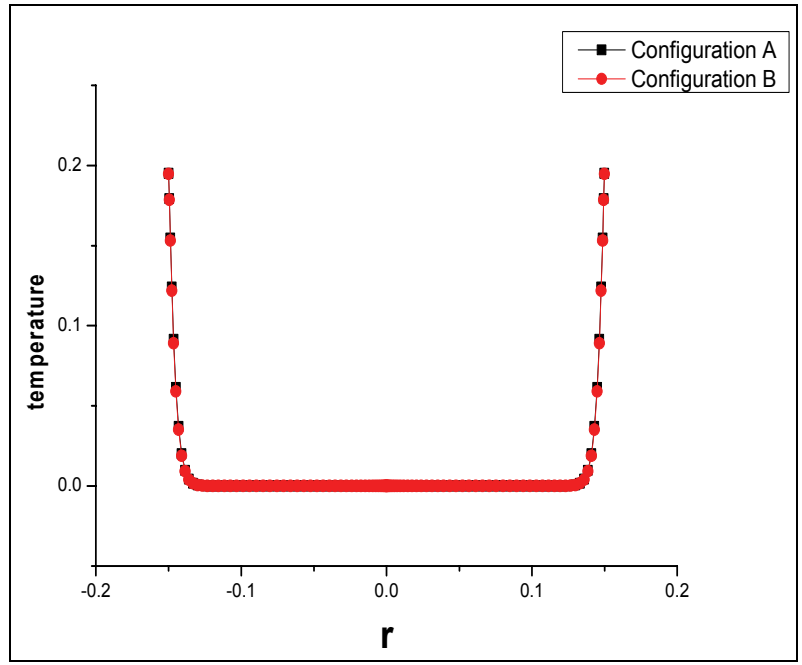

(b)

Fig. 5 Dimensionless temperature profiles for the different configurations: (a) at the entrance; (b) at the outlet.

It could be seen that the velocity increases with the increase of Rayleigh number [4].

\section{Conclusions}

Laminar, steady, viscous and Newtonian natural convection flow in a heated channel is studied in this work to improve the thermal and the dynamic qualities of a hot air generator.

This study investigates mathematically and numerically the influence of different boundary conditions through two configurations, the first one consists of a vertical cylinder with heated walls, and the second configuration is an open-ended vertical cylinder with a heating source at the entrance. 


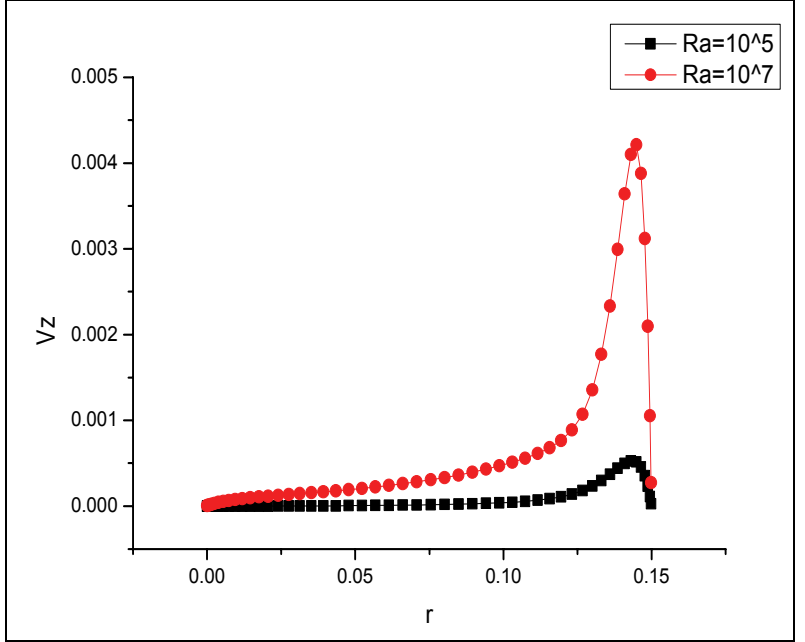

(a)

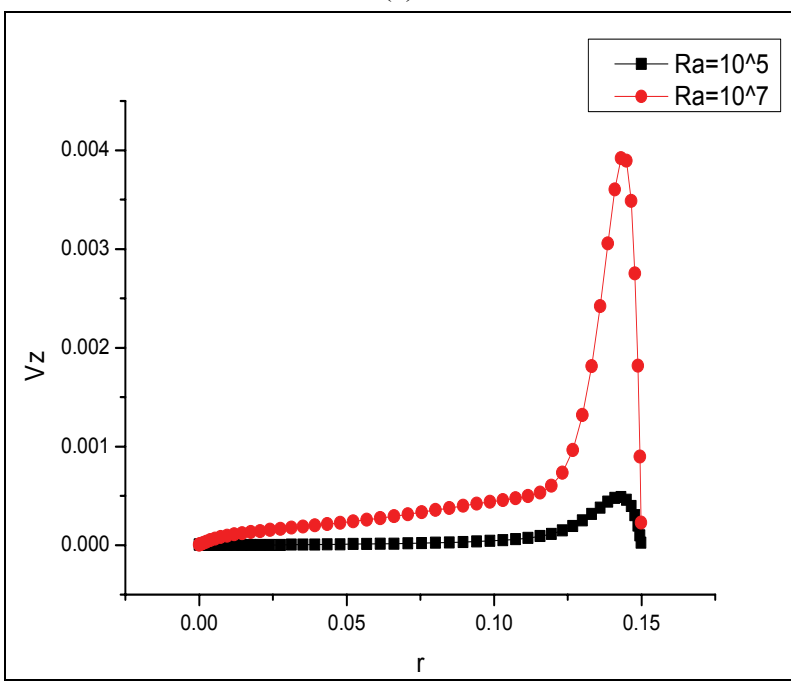

(b)

Fig. 6 Dimensionless velocity profiles for the different Rayleigh numbers: (a) at the entrance; (b) at the outlet.

Navier-Stokes equations and energy equation were solved using the finite volume method. For calculation purposes a program under FORTRAN has been implemented.

The comparison of the velocity and the temperature profiles at the generator exit permits to determine the influence of the boundary conditions and Rayleigh number on the resulting flow.

The thermal and the dynamic qualities of the flow increase with the increase of radial distance $r$ (near of the wall), while the dynamic qualities of the flow increase with the increase of Rayleigh number.

At the exit of both configurations, the flow becomes more homogenous.

\section{References}

[1] Mahmoud, A. O. M., Ben. Maad R., and Belghith, A. 1998. "Production of Hot Air with Quasi Uniform Temperature Using Concentrated Solar Radiation." Renewable Energy 13 (4): 481-93.

[2] Mahmoud, A. O. M., Zinoubi, J., and Ben. Maad R., 2007. "Study of Hot Air Generator with Quasi Uniform Temperature Using Concentrated Solar Radiation: Influence of the Shape Parameters." Renewable Energy 32: 351-64.

[3] Fontaine, J. R., Devienne, R., and Rose, S. 2006. "Numerical Simulation and Experimental Validation of Plume Flow from a Heated Disk." Numerical Heat Transfer Part A Applications 50 (7): 645-66.

[4] Versteeg, H. K., and Malalasekera, W. 2007. An Introduction to Computational Fluid Dynamics: The Finite Volume Method. Pearson Education.

[5] Kettleborough, C. F. 1972. "Transient Laminar Free Convection between Heated Vertical Plates Including Entrance Effects." International Journal of Heat and Mass Transfer 15 (5): 883-96.

[6] Ganesan, P., and Rani, H. P. 1998. "Transient Natural Convection along Vertical Cylinder with Heat and Mass Transfer." Heat and Mass Transfer 33 (5-6): 449-55. 